O artigo Admissão ao Internato Complementar em Portugal: Análise dos Resultados do Exame Nacional de Seriação entre 2006 e 2011, de Isabel Pavão Martins, foi publicado com uma gralha (Acta Med Port. 2013;26:569577).

Pelo lapso, pedimos desculpa à autora.

Na Pág. 574, tabela 3, onde se lê (assinalado a vermelho):

Tabela 3 - Análise pos hoc de Tukey HSD das notas médias no ENS por Faculdade de origem. Os grupos que se encontram na mesma coluna não diferem significativamente entre si.

\begin{tabular}{|c|c|c|c|c|c|c|c|}
\hline \multirow{2}{*}{ Faculdade de Licenciatura } & \multicolumn{7}{|c|}{ Subset for alpha $=0,05$} \\
\hline & $\mathbf{N}$ & 1 & 2 & 3 & 4 & 5 & 6 \\
\hline Faculdades Estrangeiras & 1107 & 48,85 & & & & & \\
\hline Faculdade de Ciências da Saúde da Universidade da Beira Interior & 309 & & 63,84 & & & & \\
\hline Faculdade de Medicina de Coimbra & 1597 & & 66,10 & 66,10 & & & \\
\hline Faculdade de Ciências Médicas da Universidade Nova de Lisboa & 1400 & & & 66,26 & & & \\
\hline Instituto de Ciências Biomédicas Abel Salazar & 972 & & & 67,30 & 67,30 & & \\
\hline Faculdade de Medicina de Lisboa & 1835 & & & & 69,41 & 69,41 & \\
\hline Faculdade de Medicina do Porto & 1472 & & & & & 69,95 & \\
\hline Universidade do Minho - Escola de Ciências da Saúde & 264 & & & & & 72,33 & \\
\hline Sig. & & 1,000 & 0,064 & 0,778 & 0,107 & 0,997 & 1,000 \\
\hline
\end{tabular}

deve ler-se:

Tabela 3 - Análise pos hoc de Tukey HSD das notas médias no ENS por Faculdade de origem. Os grupos que se encontram na mesma coluna não diferem significativamente entre si.

Subset for alpha $=0,05$

\title{
Faculdade de Licenciatura
}

$\begin{array}{llllllll}\mathrm{N} & 1 & 2 & 3 & 4 & 5 & 6\end{array}$

Faculdades Estrangeiras

Faculdade de Ciências da Saúde da Universidade da Beira Interior

Faculdade de Medicina de Coimbra

Faculdade de Ciências Médicas da Universidade Nova de Lisboa

Instituto de Ciências Biomédicas Abel Salazar

Faculdade de Medicina de Lisboa

Faculdade de Medicina do Porto

Universidade do Minho - Escola de Ciências da Saúde
1107

309

1597

1400

972

1835

1472

264
48,85

63,84

$66,10 \quad 66,10$

66,26

$67,30 \quad 67,30$

$69,41 \quad 69,41$

69,95

$$
\text { Sig. }
$$

$1,000 \quad 0,064$

0,778

0,107

0,997

Means for groups in homogeneous subsets are displayed. 


\section{Errata a Admissão ao Internato Complementar em Portugal: Análise dos Resultados do Exame Nacional de Seriação entre 2006 e 2011 Acta Med Port 2013:26:760-760}

Publicado pela Acta Médica Portuguesa, a Revista Científica da Ordem dos Médicos

Av. Almirante Gago Coutinho, 151

1749-084 Lisboa, Portugal.

Tel: +351218428215

E-mail: submissao@actamedicaportuguesa.com

www.actamedicaportuguesa.com

ISSN:0870-399X | e-ISSN: 1646-0758

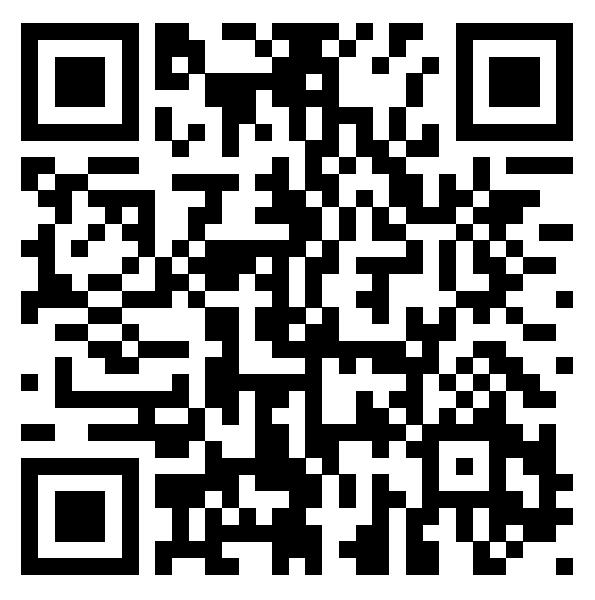

